\title{
XXXI. Note on the analysis of the rhombohedral system
}

\section{W.J. Lewis M.A.}

To cite this article: W.J. Lewis M.A. (1879) XXXI. Note on the analysis of the rhombohedral system , Philosophical Magazine Series 5, 7:42, 176-178, DOI: 10.1080/14786447908639588

To link to this article: http://dx.doi.org/10.1080/14786447908639588

曲 Published online: 13 May 2009.

Submit your article to this journal ๘

Џll Article views: 2

Q View related articles ¿ 
rotates the plane of polarization in the same direction as the other gases.

Strasburg, October 1878.

Postscript.-Since the publication of the foregoing experiments we have further improved the apparatus employed, by giving to the iron tube ( $a a$ in the figure) a length of $2 \cdot 4$ metres. The glass plates $d d$ were now so far from the ends of the six coils that, on closing a current from 70 large Bunsen elements, they did not produce any perceptible rotation. A repetition of the experiments with bisulphide-of-carbon vapour gave now also an evident rotation of the plane of polarization.

In like manner we succeeded in observing the electromagnetic rotation in gaseous sulphurous acid at $100^{\circ} \mathrm{C}$. and a pressure of about twenty atmospheres, and in sulphuretted hydrogen gas at the ordinary temperature and about twenty atmospheres.

In air, up to twenty-five atmospheres, we have not yet been able to observe any rotation. We will not omit to remark that, apart from employing very high pressures, another way presents itself of making the observations for the investigation of the rotation in air-with polarized sunlight, which with the aid of heliotropes in the direction of the earth's magnetic meridian is sent through a long stretch of the atmosphere.

XXXI. Note on the Analysis of the Rhombohedral System. By W. J. Lewis, M.A., Fellow of Oriel College, Oxford*.

7 THE methods followed by Professor Miller and most writers for obtaining the formulæ employed in determining the indices of a form in the rhombohedral system from the measured angles, or, conversely, the angles from the given indices, are, though elegant, difficult and perplexing. It occurred to me that they might be easily obtained by means of the anharmonic ratio of four poles in a zone applied to three known poles in one of the planes of symmetry, and a fourth pole whose position and indices can be directly connected with the poles of the form to be determined. This method brings out in a prominent manner the relation (2); a relation to be found in all the books, but so disguised and so little noticed as easily to be passed over, whereas from its simplicity, and from the fact that the angle involved in it is the first deduced from the ]878.

* Communicated by the Crystallological Society. Read Nov. 24, 
measured angles of a scalenohedron, it contains a smaller error than any other equation.

The figure represents the stereographic projection of some of the principal poles and planes of a rhombohedral crystal, together with the poles $\mathrm{P}$ of a form $\{h k l\}$ to be determined. The poles $r$ are $\{100\}$, $o(111)$; therefore the poles $b$ and $a$ are $\left\{\begin{array}{lll}2 & \overline{1} & \overline{1}\end{array}\right\}$ and $\left\{\begin{array}{lll}0 & 1 & \overline{1}\end{array}\right.$ respectively. Let $\mathrm{P}$ be $(h k l), \mathrm{P}_{\prime}, \mathrm{P}_{\|}$the corresponding faces repeated over $\mathrm{O} b$ and $O b_{\mu}$. Then $\mathrm{P}$, is $(h l k)$, and

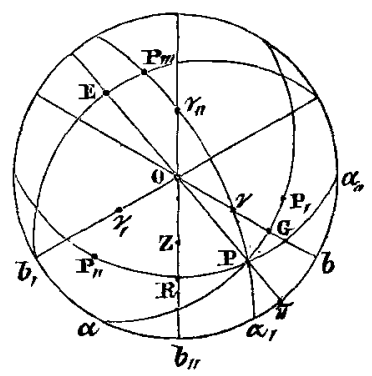
$\mathrm{P}_{\mu}(k h l)$. Let $\mathrm{Q}, \mathrm{R}, \pi$ be the intersections of the pairs of zones $[\mathrm{PP}],[\mathrm{O} b],\left[\mathrm{PP}_{/}\right]\left[\mathrm{O} b_{/}\right],[\mathrm{OP}]\left[b b_{,}\right]$respectively. Then the indices of $\mathbf{Q}$ are $(2 h, k+l, k+\bar{l})$, of $\mathbf{R}(h+k, h+k$, $2 l)$, and of $\pi(2 h-k-l,-h+2 k-l,-h-k+2 l$.

The anharmonic ratio of the poles $a, b, /, \pi, b$ gives

$$
\frac{\sin b \pi}{\sin \pi a}: \frac{\sin b b_{\prime \prime}^{\prime}}{\sin b_{, \prime} a}=\left[\frac{b \pi}{\pi a}\right]:\left[\frac{b b_{1}}{b_{1 /} a}\right]=\frac{k-l}{2 h-k-l}
$$

(Miller's 'Treatise on Crystallography,' p. 14). Hence

$$
\tan b \pi=\tan \mathrm{XOP}=\frac{(k-l) \sqrt{3}}{2 h-k-l} . \quad . \quad .
$$

The anharmonic ratio of the poles $0, r, Q, b$ gives, in a similar manner,

$$
\frac{\tan \mathrm{OQ}}{\tan \mathrm{O} r}=\left[\frac{\mathrm{OQ}}{b \mathrm{Q}}\right]:\left[\frac{\mathrm{Or}}{\bar{b} r}\right]=\frac{2 h-k-l}{2(h+k+l)} ;
$$

and writing $\mathrm{D}$ for the element $\mathrm{O} r$, we have

$$
\tan O Q=\frac{2 h-k-l}{2(h+k+l)} \tan \mathrm{D} . \quad . \quad .
$$

Similarly from the poles $\mathrm{O}, \mathrm{R}, b_{\mu}$ and $z(22 \overline{1})$ the dirhombohedral face of $r_{/ /}$we obtain

$$
\frac{\tan \mathrm{OR}}{\tan \mathrm{D}}=\left[\frac{\mathrm{RO}}{\mathrm{R} b_{j}}\right]:\left[\frac{2 \mathrm{O}}{z \bar{b}_{/ /}}\right]=\frac{h+k-2 l}{2(h+k+l)} . .
$$

From the right-angled triangle $\mathrm{POQ}$ we have

$\therefore$ from (1) and (2),

$$
\tan O P=\tan O Q \sec b \pi ; . . . .
$$

$$
\tan ^{2} \mathrm{OP}=\frac{(k-l)^{2}+(l-h)^{2}+(h-k)^{2}}{2(h+k+l)^{2}} \tan ^{2} \mathrm{D} .
$$


The equation (2) is given by Professor Miller in his ' Treatise on Crystallography,' 1839, in the form

$$
\tan \mathrm{PO} \tan \mathrm{XO} \cos \mathrm{XOP}=\frac{2 h-k-l}{h+k+l},
$$

and in the equivalent form

$$
2 \tan \mathrm{PO} \cot \mathrm{OA} \cos \mathrm{XOP}=\frac{2 h-k-l}{h+k+l},
$$

the latter being the same as (2), with the sole difference that $\tan O Q$ is replaced by its value given by equation (A). The form in which it is given by Professor Miller does not, however, bring out so prominently the simplicity and directness of the relation existing between the quantities involved in the equation and those given by observation.

As an illustration of the utility of equations (2) and $\left(2^{\prime}\right)$, let us take the determination of a scalenohedron on a mineral (such as calcite) whose elements are known. Measurement of two of the angles between adjacent faces suffices for the determination. If $\mathrm{PP}$, and $\mathrm{PP}_{1}$ are the two angles measured, we know the three sides of the triangle $a \mathrm{~Pa} a_{/ /}$; and the angle $\mathrm{P} a b=b \mathrm{Q}=\frac{\pi}{2}-\mathrm{OQ}$ is the first quantity deduced from the measurements. Equation (2) then gives a simple equation in

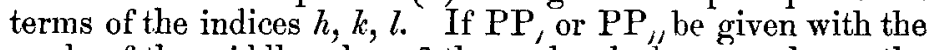
angle of the middle edge of the scalenohedron, we know the sides of the triangles $a \mathrm{P} a$ or $a_{\mu} \mathrm{Pa}$. In the first case $\mathrm{OQ}$ is determined as before, in the second $O R_{\text {; }}$ and we must employ (2) or $\left(2^{\prime}\right)$ accordingly.

To complete the analysis, I need only point out that the relations connecting the indices of dirhombohedral forms can be most simply obtained by aid of the equations connecting the indices of a face with those of the zone in which it lies. Thus $\mathrm{E}$, the face of the dirhombohedral form corresponding to $\mathrm{P}$, lies in the zones $[\mathrm{OP}]$ and $[b, \mathrm{P} / /]$, whence its indices can be at once obtained, and all the geometrical relations connecting it with $\mathrm{P}$ can be proved. Professor Maskelyne has, I believe, already given this method of deducing the indices of the dirhombohedral form in his lectures at Oxford. 